Tabelle VII

\begin{tabular}{|c|c|c|c|c|}
\hline Lichtart & $\begin{array}{c}\text { Mittlere } \\
\text { Wellenlänge } \\
\text { des Lichtes: }\end{array}$ & $\begin{array}{c}\text { Auf } l=0 \text { extrapolierte } \\
\text { relative Helligkeit } \\
\text { des } \\
\text { Ty ndall kegels : J }\end{array}$ & $J \cdot 2^{2} \cdot 10^{-8}$ & $J \cdot \lambda^{4} \cdot 10^{-14}$ \\
\hline $\begin{array}{c}\text { weiB } \\
\text { (ohne Filter }\end{array}$ & $(574 \mu \mu)^{*}$ & 1430 & - & - \\
\hline $\begin{array}{l}\text { dunkelrot } \\
\text { (Filter a) }\end{array}$ & etwa $680 \mu \mu$ & 830 & 3,84 & 1,77 \\
\hline (Filter b) & $631 \mu \mu$ & 900 & 3,58 & 1,43 \\
\hline $\begin{array}{l}\text { orangerot } \\
\text { (Filter c) }\end{array}$ & $596 \mu \mu$ & 1240 & 4,40 & 1,56 \\
\hline $\begin{array}{l}\text { olivgrün } \\
\text { (Filter d) }\end{array}$ & $566 \mu \mu$ & 1400 & 4,48 & 1,44 \\
\hline $\begin{array}{l}\text { reingrün } \\
\text { (Filter e) }\end{array}$ & $532 \mu \mu$ & 2040 & 5,78 & 1,65 \\
\hline $\begin{array}{l}\text { blaugrün } \\
\text { (Filter } f \text { ) }\end{array}$ & $484 \mu \mu$ & 2680 & 6,28 & 1,47 \\
\hline
\end{tabular}
Gleichung

* Der optische Schwerpunkt des weißen Lichtes ist berechnet nach der

$$
\lambda_{\mathrm{weiB}}=\sqrt[4]{\frac{4}{\frac{\mathrm{kMittel} \cdot 10^{-14}}{J_{\mathrm{weiB}}}}}=\sqrt[4]{\frac{1,55 \cdot 10^{-14}}{1430}}=574 \mu \mu .
$$

untersuchten Lösung abhängt, läßt sich zurzeit nicht mit Sicherheit entscheiden, da die Frage, wie sich das in den Trog eingetretene Licht in der trüben Lösung verteilt, und das Problem des sekundären Ty ndall lichtes noch offen ist.

\section{Zusammenfassung.}

In der vorliegenden Mitteilung wird über tyndallmetrische Messungen im einfarbigen Lichte berichtet.

Die Messungen, die sowohl im parallelen als auch im konvergenten Lichte durchgeführt worden sind, stehen mit der Forderung der Rayleigh'schen Theorie, dab die Menge des in einem trüben Medium zerstreuten Lichtes der vierten Potenz der Wellenlänge des Lichtes umgekehrt proportional sein soll, in befriedigender Uebereinstimmung. Weiter wird gezeigt. in welcher Weise man den optischen Schwerpunkt des weißen Lichtes bei tyndallmetrischen Untersuchungen bestimmen und wie man im weißen Lichte ausgeführte tyndallmetrische Messungen zur Besprechung der für die betreffende trübe Lösung charakteristischen Konstanten des Rayleigh'schen Gesetzes benutzen kann.

Chemisches Laboratorium

der Bergakademie Clausthal i. $H$.

\title{
Die Wirkung von Alkalisilikaten auf Ton- und Kaolinsuspensionen.
}

\author{
Von P. Rohland (Stuttgart).
}

(Eingegangen am 21. Juli 1914)

In einem der vielen Patente des Grafen Botho Schwerin ${ }^{1}$ ) findet sich als Ursache der Einwirkung von Alkalisilikaten auf Kaolin- und Tonsuspensionen folgendes angegeben:

„Die Ki eselsäure wird durch Hydrolyse in Freiheit gesetzt und vom Ton oder Kaolin a ds orbiert, während das Alkali als Elektrolyt alsbald den Solzustand hervorruft."

1). Klasse d 2 Nr. 253563 (1910).
Diese Einwirkung besteht in folgendem Vorgang: Wird $z u$ einer starken Rohkaolinsuspension eine geringe Menge einer Lösung von Alkalisilikatlösung zugesetzt, so findet eine sofortige mechanische Abscheidung der darin enthaltenen $Q u a r z$ - und $S$ andkörner statt; und zwar werden aus der Kaolinsuspension die gröberen Körner zuerst abgeschieden, dem dann die kleineren und kleinsten folgen.

Diese Quarz- und Sandkörner werden mit einiger Intensität auf den Boden des Stand- 
zylinders geschleudert, so daß auf diesem eine feste kompakte Masse sich bildet.

Die vom Grafen Botho Schwerin gegegebene, oben angeführte Erklärung hierfür ist unrichtig, wie sich leicht nachweisen läßt :

Setzt man nämlich zu einer Rohkaolinsuspension kolloide Kieselsäure, so findet keine Aus s che idu'ng der Quarz- und Sandkörner statt; auf Zusatz von Hydroxyli o ne n auch nicht.

Werden aber durch Zusatz von Alkalisilikat die Quarz- und Sandkörner abgeschieden, und setzt man dann nach entsprechender Verdünnung mit Wasser $\mathrm{OH}^{\prime}$-Ionen in genügender Konzentration hinzu, so dah der Schwellenwert, oberhalb dessen die Ausflockung der Kaolinteilchen erfolgen muh, überschritten wird, so findet auch die kolloidchemische Konstitutionsä nde rung der Ton- oder Kaolinteilchen statt ${ }^{2}$ ).

Ich habe schon damals darauf hingewiesen, daB bei dieser Ausflockung der Kaolinteilchen die Konzentration der Hydroxylionen das Maßgebende ist, und daß auch die Kationen, aber in ganz geringem Maße, diesen Vorgang beeinflussen.

Die mit dieser Ausflockung verbundene und dieser voraufgehende B e weg u $\mathrm{ng}$ in der Tonoder Kaolinsuspension, die darauf zurückzuführen ist, daß zwischen den negativ geladenen Tonoder Kaolinteilchen und den Hydroxylionen eine elektrostatische Abstoßung stattfindet, kommt in einer Kaolinsuspension, der Alkalisilikat zugesetzt worden ist, nicht vor.

2) Vgl. P. Rohland, Koll.-Zeitschr. 9, 4 (1912), über die Einwirkung von Hydroxylionen auf Kaolinemulsionen.
Und wenn zu einer sehr verdünnten Kaolinsuspension Alkalisilikat gesetzt wird, so findet, trotzdem $\mathrm{OH}^{\prime}$-Ionen in der Lösung vorhanden sind, nur eine mechanischeAbscheidung der Quarzund Sandkörner statt, aber keine Ausflockung der Kaolinteilchen, weil eben die Konzentration der OH-Ionen nicht grob genug ist, um den Schwellenwert, oberhalb dessen die Ausflockung stattfindet, $z u$ erreichen.

Diese Versuche ergeben unzweifelhaft, daB die hydrolytisch aus dem Alkalisilikat abgespaltene Kieselsäure $n i c h$ für die mechanische Abscheidung der Quarz- und Sandkörner verantwortlich gemacht werden kann, ebensowenig die abgespaltenen Hydroxylionen. Vielmehr ist als Ursache dieser schnellen Abscheidung der Quarz- und Sandkörner das nichtdissozi i ertekolloide Alkalisilikatmolekül anzusehen; hierdurch wird die Ton- oder Kaolinsuspension in einen eigentümlichen Solzustand, der eine gleichmäbig feine Verteilung der Ton- oder Kaolinteilchen herbeiführt, versetzt, so daß die Quarz- und Sandkörner, die in der Rohkaolinsuspension ohne Zusatz von Alkalisilikat haften bleiben, rasch zu Boden sinken.

Die Versuche und daraus gezogenen Schlußfolgerungen von $\mathrm{G}$. Mas ch haupt ${ }^{3}$ ) sind deshalb nicht ganz richtig, weil dieser bei der Einwirkung von Hydroxylionen auf Kaolinund Tonsuspensionen den oben beschriebenen Schwellenwert übersehen hat, worauf auch G. Wi egn e $r^{4}$ ) aufmerksam macht.

3) G. Masch ha upt, Landwirtschaftl. Versuchsstationen 83,467 (1914).

4) G. W i e g n e r, Landwirtsch. Versuchsstationen 283 (1914).

\section{Die Kolloide in Pyridin. ${ }^{1}$}

Von A. Pi eroni und E. Girardi (Bologna). (Eingegangen am 17. Juli 1914)

Die Kolloide werden in Lösungsmitteln gebildet, in welchen die betr. Körper schlecht oder gar nicht löslich sind oder, richtiger gesagt, in den gesättigten Lösungen der Kolloide selbst. Für ein und dasselbe Lösungsmittel sind die maximalen Konzentrationen, die die Körper daselbst im kolloiden Zustande erreichen können, zwar sehr verschieden, aber immer relativ gering. Ebenso wie für ein gegebenes Lösungsmittel die Maximalkonzentrationen der verschiedenen

1) Uebersetzt von J. Matula (Wien).
Kolloide verschieden sind, ebenso sind auch die maximalen Konzentrationen, die ein und dieselbe Substanz im kolloiden Zustande in verschiedenen Lösungsmitteln zeigt, verschieden, wie dies gleichfalls, wenn auch in weitaus größerem Umfange, für jene Körper gilt, die echte Lösungen geben. Zweifellos hängt diese, soweit sie die Maximalkonzentrationen der kolloiden Lösungen anbetrifft, wenigstens scheinbare UnregelmäBigkeit außer von den chemischen Eigenschaften des Lösungsmittels und der gelösten Substanz, auch von den physikalischen 